\title{
Highly Sensitive Determination of Plasma Cytokines by Time-Resolved Fluoroimmunoassay; Effect of Bicycle Exercise on Plasma Level of Interleukin-1 $\alpha$ (IL-1 $\alpha)$, Tumor Necrosis Factor $\alpha($ TNF $\alpha)$, and Interferon $\gamma($ IFN $\gamma)$
}

\author{
Hiroko Kimura, ${ }^{* 1}$ Masatoshi Suzui, ${ }^{* 2}$ Fumiko NagaO, ${ }^{* 3}$ and Kazuko Matsumoto ${ }^{* 4}$ \\ *1 Department of Forensic Medicine, Juntendo University School of Medicine, Hongo, Tokyo 113-8421, Japan \\ *2 School of Business Administration, Health Science, Meiji University, Suginami, Tokyo 168-8555, Japan \\ *3 Department of Immunology, Juntendo University School of Medicine, Hongo, Tokyo 113-8421, Japan \\ *4 Department of Chemistry, Waseda University, Okubo, Shinjuku, Tokyo 169-8555, Japan
}

\begin{abstract}
A highly sensitive time-resolved fluoroimmunoassay of human plasma cytokines is described. The cytokines such as interleukin- $1 \alpha$ (IL- $1 \alpha$ ), tumor necrosis factor $\alpha$ (TNF $\alpha$ ) are known to be acute inflammatory cytokines and it has been reported that these cytokines are secreted into blood by physical exercise. In this study, a sandwich-type immunoassay of cytokines was established using a europium chelate BHHCT-Eu ${ }^{3+}$ as a powerful labeling material. The minimum detection limits of cytokines, i.e. IL- $1 \alpha$, TNF $\alpha$, and interferon $\gamma$ (IFN $\gamma$ ) were about $1 / 10$ smaller than those of enzymelinked immunosorbent assay currently used. By this immunoassay we investigated cytokine increase/decrease in plasma which was thought to derive from the myocytes damaged by bicycle exercise. Healthy young men performed two kinds of bicycle ergometer exercises, under conditions of an incremental and a constant loading. Blood samples were taken before, during, and after exercises, and the concentration levels of plasma IL- $1 \alpha$, TNF $\alpha$, and IFN $\gamma$ were determined. In the case of incremental exercise, IL- $1 \alpha$ increased significantly at the first stage but decreased to the basal level from the second stage, in spite of heavier exercise. In the case of $30 \mathrm{~min}$ constant exercise, the level of plasma IFN $\gamma$ increased in recovery period, $2 \mathrm{~h}$ after the light-exercise. TNF $\alpha$ level was significantly higher in a heavy-exercise. The concentration of IL- $1 \alpha$ peaked at the early stage of the incremental exercise; this fact has not been reported in previous studies. This cytokine is unique in showing a sudden increase during the early stage, while others increase after the exercise. Our highly sensitive assay made it possible to detect a slight change in plasma cytokines.
\end{abstract}

(Received December 25, 2000; Accepted February 13, 2001)

It has been reported that physical exercise induces various changes in the human immune system. ${ }^{1}$ For example, strenuous exercise induces an increase in inflammation while moderate walking reduces it. ${ }^{2}$ At present in our society, the number of the elderly is increasing and many of them are very interested in "physical exercises for healthy life". In the human natural immune system, natural killer (NK) cells and cytokines are well known as important components of the host defense system. NK cells are lymphocytes that kill certain types of tumor cells and virally infected cells without prior sensitization or restriction by the major histocompatibility complex. ${ }^{3,4}$ It is believed that NK cells have a role in immune surveillance as a primary defense mechanism against cancers or virally induced diseases. $^{5-7}$ Cytokines are termed proteins of low molecular weight that stimulate or inhibit the differentiation, proliferation or function of immune cells. They are divided roughly into two types, i.e. immunoregulatory and inflammatory cytokines. Among these cytokines, interleukin-1 (IL-1), IL-6, and tumor necrosis factor $\alpha$ (TNF $\alpha$ ) are known to be acute inflammatory cytokines, and it is reported that these cytokines are also secreted into the blood by physical exercise. ${ }^{8}$ Cannon and Kluger $^{9}$ first reported the relation between exercise and cytokine. In their report, endogenous pyrogen activity in human plasma after exercise was suggested from the fact that a rat's body temperature rose by incubation with human plasma after exercise. In those days, it was considered that this phenomenon was induced by IL-1, but it was later pointed out by Bagby et $a l .{ }^{10}$ that the biological assay used in the early studies could not distinguish IL-1 and IL-6, and they suggested IL-6 as a pyrogenic substance. In 1992, Sprenger et al. ${ }^{11}$ reported that levels of IL-1, IL-6, TNF $\alpha$, IFN $\gamma$ elevated in urine of runners covering a distance of $20 \mathrm{~km}$ within $2 \mathrm{~h}$, and this proved IL-1 increase after an exercise. It is also reported that, except for IL6 , cytokines were not reliably detected in plasma but were present in urine instead. In 1994 , Ullum et al. ${ }^{12}$ reported that plasma IL- 6 was enhanced by bicycle exercise but IL- $1 \alpha$, IL$1 \beta$, and TNF $\alpha$ were not. In the report they say that levels of plasma IL- $1 \alpha$, IL- $1 \beta$ and TNF $\alpha$ were below the detection limit of their enzyme-linked immunosorbent assay (ELISA) in most subjects. More recently Ostrowski et al. ${ }^{8}$ reported an increase of IL- $1 \beta$, IL- 1 receptor antagonist (IL-1ra), IL-6, and TNF $\alpha$ in plasma in strenuous exercise (in the Copenhagen marathon race), measured by ELISA. The study suggested that the increase in these cytokines was an inflammatory response to myocyte damage during the exercise.

In this study, a sandwich-type time-resolved fluoroimmunoassay (TR-FIA) of cytokines was established using a europium chelate, i.e. 4,4'-bis $\left(1^{\prime \prime}, 1^{\prime \prime}, 1^{\prime \prime}, 2^{\prime \prime}, 2^{\prime \prime}, 3^{\prime \prime}, 3^{\prime \prime}\right.$ - 
heptafluoro-4",6"-hexanedion-6"-yl)-chlorosulfo-o-terphenyl (BHHCT)-Eu ${ }^{3+}$ as a powerful labeling material. In 1998, Yuan et al. reported the synthesis of a new tetradentate chlorosulfonyl $\beta$-diketone type europium chelate, (BHHCT)-Eu ${ }^{3+}$, and its application as a fluorescent label in the TR-FIA revealed the high sensitivity of the method. ${ }^{13}$ By this immunoassay, we investigated cytokine increase/decrease in plasma thought to derive from myocyte damage caused by bicycle exercises. Healthy young men performed two kinds of ergometer bicycle exercises, i.e. an incremental and a constant loading. In our report, the relation between strength of exercises and levels of cytokines was discussed, and changes in the cytokine levels (pre-exercise and post-exercise levels of plasma IL-1 $\alpha$, tumor TNF $\alpha$ and IFN $\gamma$ ) were also demonstrated. Furthermore, a new result was obtained: that the concentration of IL- $1 \alpha$ peaked at the early stage of the incremental loading exercises.

\section{Experimental}

\section{Antibodies and antigens}

Purified mouse anti-human IL- $1 \alpha$, anti-IFN $\gamma$, anti-TNF $\alpha$ were obtained from PharMingen (San Diego). Biotinylated mouse anti-human IL-1 $\alpha$, anti-IFN $\gamma$, and anti-TNF $\alpha$ were also obtained from PharMingen (San Diego). Recombinant human IL- $1 \alpha$, human IFN $\gamma$, and human TNF $\alpha$ from PharMingen (San Diego) were used as standards. Human IL- $1 \alpha$ and IFN $\gamma$, ELISA system kits were purchased from Amersham International (Buckinghamshire).

\section{Labeling for streptavidin}

BHHCT was synthesized according to the method employed in our previous article. ${ }^{13}$ Streptavidin (SA) was obtained from Chemicon International Inc. (Temecula, CA). A streptavidin and bovine serum albumin (BSA) conjugate was prepared as reported in the previous article. ${ }^{14}$ BHHCT was labeled to the SA and BSA conjugate. ${ }^{14}$ It was diluted to $1: 700$ by adding $0.05 \mathrm{M}$ Tris- $\mathrm{HCl}$ buffer $(\mathrm{pH} 7.8)$ containing $1.0 \times 10^{-7} \mathrm{M} \mathrm{EuCl}_{3}$, $0.2 \% \mathrm{BSA}, 0.1 \% \mathrm{NaN}_{3}$, and $0.9 \% \mathrm{NaCl}$, and was reacted at $56^{\circ} \mathrm{C}$ for $2 \mathrm{~h}$ before use in immunoassay.

\section{Time-resolved fluoroimmunoassay (TR-FIA) of cytokines}

Sandwich type immunoassay was employed. Monoclonal anti-IL- $\alpha$, anti-IFN $\gamma$, and anti-TNF $\alpha$ were diluted with $0.1 \mathrm{M}$ carbonate-bicarbonate buffer ( $\mathrm{pH} 9.3$ ) to $2 \mu \mathrm{g} / \mathrm{mL}$. Diluted antibodies $(200 \mu \mathrm{g} / 100 \mathrm{ml})$ of each were used for coating wells of the microtiter plates (Fluoro Nunc module plate, Nalge Nunc International). After washing the wells twice with $0.05 \mathrm{M}$ Tris-HCl buffer ( $\mathrm{pH} 7.8$ ) containing $0.05 \%$ Tween 20 and once with Tris- $\mathrm{HCl}$ buffer, the plates were stored at $-20^{\circ} \mathrm{C}$ until use. To the coated wells, $50 \mu \mathrm{l}$ of diluted standard cytokine solutions or serum samples (diluted to 2 - 5 times with Tris- $\mathrm{HCl}$ buffer) were added and these were incubated for $1 \mathrm{~h}$ at room temperature. Then $50 \mu \mathrm{l}$ of corresponding biotinylated antibodies $(1 \mu \mathrm{g} / \mathrm{mL})$ were added. After incubation for $1 \mathrm{~h}$ at room temperature, the wells were washed 3 times with Tris- $\mathrm{HCl}$ buffer containing Tween 20 , and then a $50 \mu \mathrm{l}$ volume of SABSA-BHHCT-Eu ${ }^{3+}$ was added. After incubation for $2 \mathrm{~h}$ at $37^{\circ} \mathrm{C}$, the wells were washed 4 times with $0.05 \mathrm{M}$ Tris- $\mathrm{HCl}$ buffer ( $\mathrm{pH}$ 9.1), and the solid-phase fluorescence was measured. For the fluorescence measurement, Wallac ARVO sx multi label counter (Amersham Pharmacia Biotech) was used with the measurement conditions of delay time of $0.2 \mathrm{~ms}$, window time of $0.4 \mathrm{~ms}$, excitation of $340 \mathrm{~nm}$, and the measurement wavelength of $615 \mathrm{~nm}$.
Table 1 Physical properties of subjects

\begin{tabular}{ccccc}
\hline Subject & Age (years) & Height $/ \mathrm{m}$ & Body mass $/ \mathrm{kg}$ & $\%$ Fat, \% \\
\hline Exercise 1 & $19 \pm 1.3$ & $1.6 \pm 0.07$ & $63 \pm 5.7$ & $17 \pm 1.6$ \\
Exercise 2 & $20 \pm 1.7$ & $1.6 \pm 0.07$ & $63 \pm 5.2$ & $16 \pm 1.8$ \\
\hline
\end{tabular}

Enzyme-linked immunosorbent assay (ELISA) of IL-1 $\alpha$ and IFN $\gamma$ ELISA of IL- $1 \alpha$ and IFN $\gamma$ were performed according to the directions of their kits. Basically, procedures of the assays were the same as the TR-FIA. Instead of SA-BSA-BHHCT-Eu ${ }^{3+}$, $100 \mu 1$ of horse-raddish-peroxidase conjugated SA and $100 \mu$ lof substrate reagent (tetramethylbenzidine) were added and after addition of $100 \mu \mathrm{l}$ of stop solution ( $0.18 \mathrm{M}$ sulfuric acid), absorbance at $450 \mathrm{~nm}$ was measured.

\section{Subjects and exercise protocols}

Five (for experiment 1 ) or 6 (for experiment 2) healthy young men who do not smoke participated in the study. All subjects were informed about the purpose and risks of the study, before written informed consent was obtained. For each subject, maximum oxygen consumption ( $\dot{\mathrm{V} O} 2 \mathrm{max}$ ) was determined prior to the experiment. $\mathrm{V} O 2$ max was determined as follows. Subjects pedaled on the same cycle ergometer as used in the experiments at the pedal cadence of 80 revolutions/min and were loaded incrementally ( $30 \mathrm{watt} / \mathrm{min}$ ). The expired breath of each subject was collected and the concentrations of $\mathrm{O}_{2}$ and $\mathrm{CO}_{2}$ were analyzed. ${ }^{15}$ Physical properties of subjects are shown in Table 1. Subjects were allowed to drink water and to eat a 400 kcal diet at seven in the morning but not allowed to take medicine and alcohol in the evening before the experiment. They had been well rested and refrained from exhaustive training on the day before the experiment. The experiments consisted of 2 different exercises on a bicycle ergometer. Exercise 1 was incremental 4 steps loading at $80 \mathrm{rpm}$ (30, 50, 70 , and $80 \%$ VंO2max). Exercise 2 was constant loadings with two different \% $\dot{V} O 2 m a x$ (50 and $70 \%$ VंO2max). Subjects were catheterized at the forearm and after 5 min warm-up exercise they started proper exercise.

\section{Blood sampling}

In exercise 1, blood was taken before and after the warm-up, and at the end of each stepwise loading exercise during $5 \mathrm{~min}$. In exercise 2, blood was taken before the warm-up, $5 \mathrm{~min}, 60$ $\mathrm{min}$, and $120 \mathrm{~min}$ after finishing the exercise. All experiments were performed in a room conditioned at $20-25^{\circ} \mathrm{C}$ and $50-60$ $\%$ humidity. Plasma was separated from blood and was stored at $-80^{\circ} \mathrm{C}$ until use.

\section{Correction for plasma volume and statics}

Changes in plasma volume were calculated from hemoglobin concentration and hematocrit value according to the method reported by Dill and Costill, ${ }^{16}$ and cytokine concentrations were corrected. Variances in the obtained concentration values were analyzed by ANOVA $(p<0.05)$ and statistical calculations were performed by Bonferroni's post hoc test $(p<0.05)$ using StatView (Abacus Concepts Inc.).

\section{Results and Discussion}

\section{The calibration curve of cytokines}

The calibration curves of IL- $1 \alpha$, IFN $\gamma$, and TNF $\alpha$ are shown in Figs. 1A, 1B and 1C. In our TR-FIA, the minimum detection 

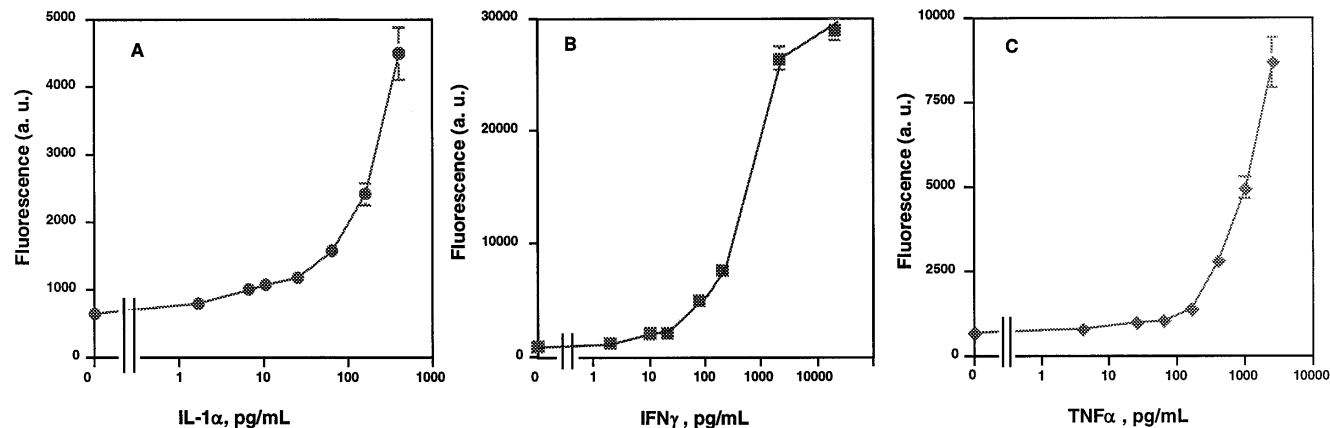

Fig. 1 Calibration curves of IL- $1 \alpha$, IFN $\gamma$, and TNF $\alpha$ determined by TR-FIA. Assay points represent mean values $( \pm \mathrm{SD})$ of four independent determinations.

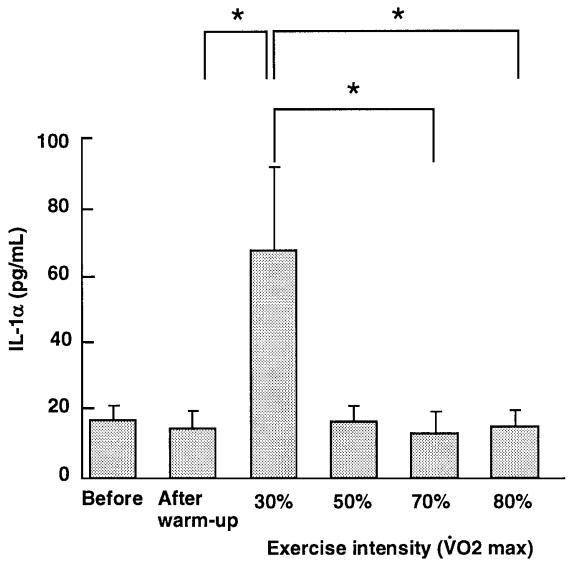

Fig. 2 Changes in plasma IL- $1 \alpha$ levels during incremental exercise. Data are means $\pm \mathrm{SD}$ of 5 individuals. *A pair with significant difference $(p<0.05)$.

limits of these cytokines are IL- $1 \alpha: 1.5 \mathrm{pg} / \mathrm{ml}$, IFN $\gamma: 2 \mathrm{pg} / \mathrm{ml}$ TNF $\alpha: 5 \mathrm{pg} / \mathrm{ml}$. The detection limit of the present method was defined by using $3 \times$ standard deviation (SD) of the background, as reported in the previous report. ${ }^{17}$ Recoveries were obtained by adding $25.6 \mathrm{pg} / \mathrm{mL}$ and $160 \mathrm{pg} / \mathrm{mL}$ of standard recombinant human IL- $1 \alpha$ to human sera whose IL- $1 \alpha$ level $(31.2 \mathrm{pg} / \mathrm{mL})$ was already determined. Recoveries of IFN $\gamma$ and IL- $1 \alpha$ were obtained by the same method using recombinant standard antigens and human sera containing $36.2 \mathrm{pg} / \mathrm{mL}$ of IFN $\gamma$ and $25.4 \mathrm{pg} / \mathrm{mL}$ of TNF $\alpha$. The obtained result showed $101,96 \%$ for IL- $1 \alpha, 91.0,95.6 \%$ for IFN $\gamma$, and $104,106 \%$ for TNF $\alpha$. For the intra-assay, the coefficients of variation were 1.2 to $8.4 \%$ for IL- $1 \alpha$ (at 8 different concentrations), 1.9 to $4.2 \%$ for IFN $\gamma$ (at 7 different concentrations), and 2.7 to $8.2 \%$ for TNF $\alpha$ (at 8 different concentrations). These minimum detection limits are $1 / 14$ to $1 / 10$ smaller than those of ELISA kits currently used, and quantifiable ranges were wide enough to determine the cytokine concentrations of plasma samples.

\section{Levels of plasma IL-I $\alpha$ and IFN $\gamma$ during incremental loading} exercise assayed by TR-FIA

The levels of plasma IL- $1 \alpha$ and IFN $\gamma$ during incremental exercise are shown in Figs. 2 and 3. Significant differences between each stage are marked. The level of IL- $1 \alpha$ increased significantly at the first stage of the incremental exercise and then decreased to the basal level in spite of heavier exercise. The concentration of IL- $1 \alpha$ peaked at the early stage of the exercise; this fact has not been reported in previous studies.

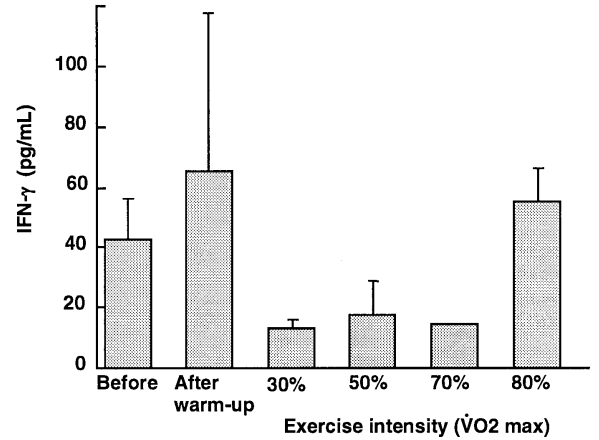

Fig. 3 Changes in plasma IFN $\gamma$ levels during incremental exercise. Data are means \pm SD of 5 individuals.

Until now, IL- 6 but not IL- $1 \alpha$ or TNF $\alpha$ has been found in plasma following concentric bicycle exercise. ${ }^{12}$ IL- $\beta$ and IL-1ra have been reported to increase after exercises, ${ }^{8}$ but there is no report stating IL- $1 \alpha$ increase. This is probably because the IL$1 \alpha$ level is slight in plasma. ${ }^{12}$ But our highly sensitive TR-FIA made it possible to detect a slight change in plasma cytokines. This cytokine is different from other cytokines in that it shows a sudden increase at the early stage while others increase after the exercise. But it is possible that this increase might be caused by other factors than myocyte damage. Further investigations concerning the mechanisms will be necessary and they are now under study. As for plasma IFN $\gamma$ during incremental exercises, its level did not change significantly (Fig. 3).

Levels of plasma IL-1 $\alpha$, IFN $\gamma$ and TNF $\alpha$ after constant exercise assayed by TR-FIA

Figures $4-6$ show the changes in cytokine levels after $30 \mathrm{~min}$ constant exercises of two different $\dot{\mathrm{VO}} 2$ max. As shown in the figures, the levels of cytokines just before and $2 \mathrm{~h}$ after exercise are compared. (The details of change in time lapse after exercise will be reported in a later article). The level of IL- $1 \alpha$ after light constant exercise $(50 \% \dot{\mathrm{V} O} 2 \mathrm{max})$ did not change significantly. In heavy-exercise $(70 \% \dot{\mathrm{V} O} 2 \mathrm{max})$, significant change in time lapse after exercise was observed (significant ANOVA interaction was observed) but no significant difference from basal level was observed at any point beyond a period of $2 \mathrm{~h}$.

As shown in Fig. 5, in light-exercise, after 2 h' recovery time the level of plasma IFN $\gamma$ increased from the level before exercise $(p<0.05)$. In heavy-exercise, the level decreased during $2 \mathrm{~h}$ recovery time (significant ANOVA interaction was observed) but no significant difference from basal level was observed at any point over a period of $2 \mathrm{~h}$. The increase may 


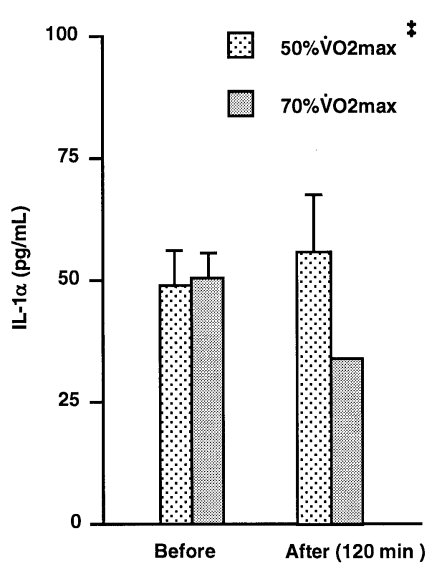

Fig. 4 Changes in plasma IL- $1 \alpha$ levels after constant exercise. Data are means \pm SD of 6 individuals. $\ddagger$ Significant ANOVA interaction $(p<0.05)$.

come later than in light-exercise. When the IFN $\gamma$ level was compared in light-exercise and heavy-exercise, it was higher in light-exercise.

As shown in Fig. 6, in heavy-exercise TNF $\alpha$ level significantly increased from basal level. Its level was significantly higher at every point than in light-exercise. As TNF $\alpha$ is thought to cause muscle proteolysis, ${ }^{18}$ heavier exercise with skeletal muscle damage induces a higher level of plasma TNF $\alpha$. TNF $\alpha$ level, when compared between light-exercise and heavy-exercise, peaked later in heavy-exercise $(60 \mathrm{~min})$ than in light-exercise $(5 \mathrm{~min})$, but no significant difference was observed.

Until now, it has been considered that the increase in cytokines after exercise is mainly induced by damages to myocytes. This supposition is based on the following findings: First, pre-mRNA for IL- $1 \alpha$, IL- $1 \beta$, IL-6, and TNF $\alpha$ was detected in blood mononuclear cells, but these cytokines' amounts in plasma did not change in relation to exercise. ${ }^{12}$ From this, Ullum et al. concluded that the increase in plasma cytokines was not caused by the monocytes increase after exercise. ${ }^{12}$ But they admit that the levels were not high enough to be detected by their assay. Secondly, after exercise, IL-6 mRNA was detected in biopsied muscle, which was not found before. ${ }^{19}$ Thirdly, TNF $\alpha$ levels increased after hard exercises which involve such contractions and expansions of muscles as occur during hill running, and which give more damage to muscle fibers. $^{18,20}$ In our study, TNF $\alpha$ increased in heavier exercises, and this supports the general supposition that this cytokine is produced by damaged myocytes.

As to IFN $\gamma$, it is said that excessive exercise suppresses IFN $\gamma$ production and as a result it causes the transit immunosuppression. ${ }^{21,22}$ In our result, IFN $\gamma$ increased during 2 h' recovery time in light-exercise, but in heavy-exercise it did not increase after exercise. It is possible that in heavy-exercise an increase comes later or it may not come at all. Further investigations will be necessary.

Although release of cytokines is considered to differ according to kinds of exercise and their duration, Ullum et al. ${ }^{12}$ reports, as it was mentioned above, that IL- $1 \alpha$ and TNF $\alpha$ were not detected in serum after bicycle exercises similar to ours. They say, as one of the possible causes, that sensitivity of their ELISA was not high enough to detect these cytokines. In the recent measurement using high sensitivity ELISA kits, ${ }^{8}$ Ostrowski et al. say that TNF $\alpha$ was detected in serum samples

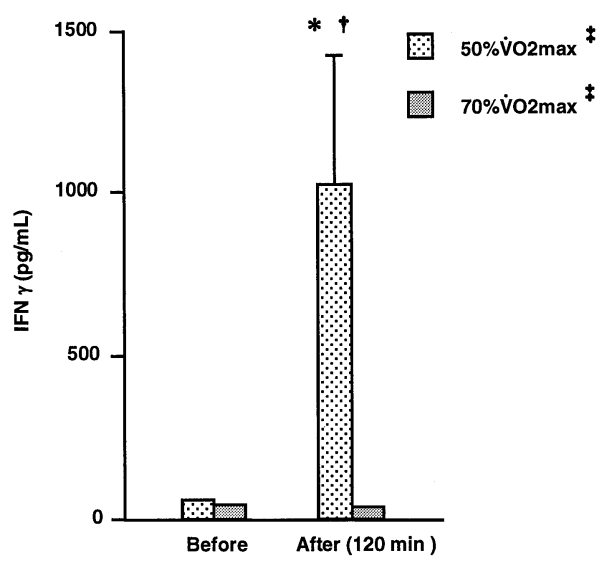

Fig. 5 Changes in plasma IFN $\gamma$ levels after constant exercise Data are means \pm SD of 6 individuals. *Significant increase in lightexercise $(p<0.05)$. $\dagger$ Significant difference after exercise $(p<0.05)$ : light-exercise $v s$. heavy-exercise. $\ddagger$ Significant ANOVA interaction $(p<0.05)$.

after marathon race, but IL- $1 \alpha$ was not assayed in spite of the fact that IL- $1 \beta$ and cytokine inhibitor, IL-1ra were detected. It was described in their report that cytokines like IL-1 may be produced locally and may be rapidly cleared from the circulation. Our sensitive TR-FIA has detected IL- $1 \alpha$ in plasma during exercise which was not found in previous studies. As far as we know, this is the first study to report a sudden increase of IL- $1 \alpha$ at the early stage of exercise. This increase is not caused by myocyte damages but may be caused by other factors like an exothermic reaction which is one of the functions of IL- $1 .{ }^{23}$ It is true that exercise raises body temperature, but in our opinion what raises the temperature is not exercise itself but probably IL-1 exothermic activity which prepares bodily conditions suitable to physical exercise. This may explain the increase at the early stage of exercise.

Cytokines are known to enhance the expression of various adhesion molecules of hemal endothelial cells. The increase or decrease of cytokines is considered to correlate to the modulation of lymphocytes including NK cells via enhanced adhesion molecules. ${ }^{24}$ On the other hand, it is considered that the modulation of NK-cell activity (NK cell cytotoxicity) or NK cell numbers, which is induced by exercise, stimulates the immune system or causes immune deficiency. ${ }^{24}$ For the promotion of physical activities and public health, further research on the effect of exercises on the immune systems is necessary. The assay of cytokines in plasma which affect immune systems should be of great help for these purposes.

It is reported that the cytokine response to exercise has similarities to that observed after trauma. ${ }^{25}$ Since cytokine assay is conventionally employed in post-operative treatments, our TR-FIA of cytokines should be applicable to forensic investigations such as those of wound age determination.

\section{Acknowledgements}

This study was supported by CREST (Core Research for Evolutional Science and Technology) of Japan Science and Technology Corporation (JST) and also by the Meiji Life Insurance Company, Tokyo, Japan. The authors thank Dr. Tomohiro Kodama and Dr. Nobuhiro Tsukada for expert assistance throughout the experimental procedure. 




Fig. 6 Changes in plasma TNF $\alpha$ levels after constant exercise Data are means \pm SD of 6 individuals. * Significant increase in heavy-exercise $(p<0.05)$. $†$ Significant difference after exercise $(p<$ $0.05)$ : light-exercise $v s$. heavy-exercise.

\section{References}

1. J. G. Cannon, J. Appl. Physiol., 1993, 74, 973.

2. D. C. Nieman, Can. J. Physiol. Pharmacol., 1998, 76, 573.

3. G. Trinchieri, Adv. Immunol., 1989, 47, 187.

4. C. Lopez, D. Kirkpatrick, S. Read, P. Fitzgerald, J. Pitt, and S. Pahwa, J. Infect., 1983, 197.

5. M. Jondal and H. Pross, Int. J. Cancer, 1983, 15, 596.

6. R. B. Herberman and J. R. Ortaldo, Science, 1981, 214, 24.

7. J. E. Talmadge, K. M. Meyers, D. J. Prieur, and J. R. Starkey, Nature, 1980, 284, 622.

8. K. Ostrowski, T. Rohde, S. Asp, P. Schjerling, and B. K. Pedersen, J. Physiol., 1999, 515, 287.

9. J. G. Cannon and M. J. Kluger, Science, 1983, 220, 617.
10. G. J. Bagby, L. D. Crouch, and R. E. Shepherd, "Exercise and Immune Function", ed. G. L. Hoffman, 1996, CRC Press, New York, 55.

11. H. Sprenger, C. Jacobs, M. Nain, A. M. Gressner, H. Prinz, W. Wesemann, and D. Gemsa, Clin. Immunol. Immunopathol., 1992, 63, 188.

12. H. Ullum, P. M. Haahr, M. Diamant, J. Palmo, J. HalkjaerKristensen, and B. K. Pedersen, J. Appl. Physiol., 1994, 77, 93.

13. J. Yuan, K. Matsumoto, and H. Kimura, Anal. Chem., 1998, 70, 596.

14. J. Yuan, G. Wang, H. Kimura, and K. Matsumoto, Anal. Biochem., 1997, 254, 28.

15. M. Suzui, F. Nagao, K. Takeda, H. Yagita, and K. Okumura, "Research-Aid Report (in Japanese)", Meiji Life Foundation of Health and Welfare, 1999, 14, 76.

16. D. B. Dill and D. L. Costill, J. Appl. Physiol., 1974, 37, 247.

17. J. Yuan, G. Wang, H. Kimura, and K. Matsumoto, Anal. Sci., 1998, 14, 421 .

18. M. D. Nawabi, K. P. Block, and M. C. Chahrabarti, J. Clin. Invest., 1990, 85, 256.

19. K. Ostrowski, T. Rohde, M. Zacho, S. Asp, and B. K. Pedersen, J. Physiol., 1998, 508, 949.

20. G. T. Espersen, A. Elbaek, and A. Ernst, APMIS, 1990, 98 , 395.

21. M. L. Kohut, J. M. Davis, D. A. Jackson, P. Jani, A. Ghaffar, E. P. Mayer, and D. A. Essig, Am. J. Physiol., 1998, 275, 1089.

22. H. Northoff, A. Berg, and C. Weinstock, Can. J. Physiol. Pharmacol., 1998, 76, 497.

23. J. G. Cannon, W. J. Evans, V. A. Hughes, C. N. Meredith, and C. A. Dinarello, J. Appl. Physiol., 1986, 61, 1869.

24. J. Rehman, P. J. Mills, S. M. Carter, J. Chou, J. Thomas, and A. S. Maisel, Brain Behav. Immun., 1997, 11, 343.

25. K. Ostrowski, C. Hermann, A. Bangash, P. Schjerling, J. N. Nielsen, and B. K. Pedersen, J. Physiol., 1998, 513, 889. 